\title{
Aquablation among novice users in Canada: A WATER II subpopulation analysis
}

\author{
Kevin C. Zorn ${ }^{1}$; S. Larry Goldenberg ${ }^{2}$; Ryan Paterson²; Alan So ${ }^{2}$; Dean Elterman ${ }^{3}$; Naeem \\ Bhojani $^{1}$ \\ ${ }^{1}$ Division of Urology, University of Montreal Hospital Centre, Université de Montréal, Montreal, QC, \\ Canada; ${ }^{2}$ Department of Urologic Sciences, University of British Columbia, Vancouver, BC, Canada; ${ }^{3}$ Department \\ of Urology, University of Toronto, University Health Network, Toronto, ON, Canada
}

Funding: The study was funded by PROCEPT BioRobotics.

Cite as: Can Urol Assoc J 2018 October 15; Epub ahead of print. http://dx.doi.org/10.5489/cuaj.5501

Published online October 15, 2018

$* * *$

\section{Abstract}

Introduction: Surgical management options for lower urinary tract symptoms/benign prostatic hyperplasia (LUTS/BPH) associated with prostates of small to moderate volume (<80 cc) are numerous, however, for men with enlarged prostates ( $>80 \mathrm{cc}$ ), many of these options are neither safe nor effective. Recently, Aquablation (PROCEPT BioRobotics, Inc., U.S.), a precise ultrasound-guided, robotically executed prostatic ablative procedure, has become available with U.S. Food and Drug Administration approval. Herein, we present three-month safety and efficacy data from the cohort of Canadian men included in the WATER II trial.

Methods: WATER II is a prospective clinical trial of the Aquablation system for the treatment of BPH in men with prostate volumes between 80 and 150 cc. Only patients from Canada were included for this analysis. At baseline, subjects completed International Prostate Symptom Score (IPSS), as well as several validated questionnaires, uroflowmetry and post-void residual volume measurements, and underwent standard laboratory blood assessment. These were repeated at one and three months post-Aquablation.

Results: A total of 19 subjects who met inclusion and exclusion criteria were enrolled at three Canadian academic sites. Mean pre-, one-month, and three-month post-treatment IPSS scores

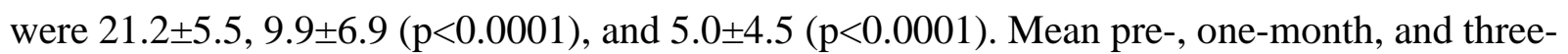
month post-treatment maximum urinary flow rates (Qmax) were $6.6 \pm 3.1 \mathrm{ml} / \mathrm{s}, 19.5 \pm 6.1 \mathrm{ml} / \mathrm{s}$ $(\mathrm{p}<0.0001)$, and $23.1 \pm 9.2 \mathrm{ml} / \mathrm{s}(\mathrm{p}<0.0001)$. The Clavien-Dindo grade 2 or higher event rate at three months was $31.6 \%$ (six events).

Conclusions: In this short-term, three-month analysis of Canadian men, Aquablation appears to provide a strong surgical alternative in patients with LUTS/BPH due to larger prostate volumes, 
with impressive functional outcomes, relatively short operative time and length of hospital stay, and acceptable complication and low transfusion rates.

\section{Introduction}

Lower urinary tract symptoms secondary (LUTS) to benign prostatic hyperplasia (BPH) increases significantly with age[1]. The occurrence of moderate-to-severe LUTS occurs in about one quarter of men in their 50s, one third of men in their 60s, and about half of all men 80 years or older[2]. While LUTS/BPH is often not life threatening, it can have a significant impact on quality of life and, when left untreated, may lead to serious complications such as infection, calculus formation, bleeding, urinary retention, and deterioration of renal function[2]. Although medical therapy (alpha1-selective adrenergic receptor antagonists and in-combination with 5alpha-reductase inhibitors for larger prostates) has remained the cornerstone of BPH therapy, undesired side-effect profiles, lifetime drug commitment and progression of LUTS must be considered during patient counseling[3].

Surgical management options for LUTS/BPH associated with prostates of small to moderate volume ( $<80 \mathrm{cc}$ ) are numerous and include traditional transurethral resection and laser endoscopic techniques (vaporization/enucleation), with varying success rates[2, 4]. However, for men with significantly enlarged prostates (>80 cc), many of these options are neither safe nor efficacious and not recommended per CUA/EAU/AUA guidelines[4-6]. Although not commonly performed in the modern era with access to less invasive alternatives, open simple prostatectomy (OSP) is an appropriate and effective treatment alternative for large prostates. OSP is the most invasive surgical method requiring not only abdominal-wall access, but longer hospitalization and catheterization with reported transfusion rates between 7-24\%[7-10]. Long-term complications include transient urinary incontinence (8-10\%), bladder neck contracture and urethral stricture (5-6\%)[4, 7, 8]. Moreover, GreenLight XPS (Boston Scientific, Marlborough, MA, USA) photoselective vaporization (PVP) has been well studied as an alternative in large prostates, particularly for anticoagulated men[11], however risks of higher retreatment rates and need for conversion to transurethral resection due to intra-operative bleeding have been observed[11, 12]. In addition, unless ejaculatory duct preservation is performed for PVP, most if not all patients will have retrograde ejaculation regardless of the modality that is used.

Finally, Holmium enucleation of the prostate (HoLEP) has been extensively studied in RCTs to OSP for large prostates demonstrating significant and durable improvements in all voiding parameters, IPSS, and PSA reduction and can be used to treat men on anticoagulation and those with bleeding dyscrasia[13-15]. There is a low reoperation rate (approximately $4 \%$ for recurrent LUTS) with long follow-up (up to 7-8 years)[16]. Unfortunately, despite compelling data, HOLEP has not been widely adopted largely due to a steep learning curve (estimated $>20-50$ cases)[16] often requiring fellowship training. Operating time, difficulty of the enucleation and need for intra-vesical morcellation seem the most important hurdles for a beginner[17].

More recently, Aquablation (PROCEPT BioRobotics, Inc., USA), a robotically executed, surgeon-guided, ultrasound-imaging aided, waterjet treatment has become available with FDA approval in December 2017 as an option for the management of LUTS/BPH[18]. In short, 
Aquablation therapy is the only treatment for BPH that combines the three key elements to remove prostate tissue safely, quickly and precisely, namely 1) real-time, multi-dimensional imaging for improved decision-making and treatment planning, 2) accuracy of an autonomous robot for precise treatment execution according to the surgeon's plan and 3) power of a heat-free waterjet, which eliminates the possibility of complications arising from thermal injury. In a randomized, double-blinded trial of Aquablation vs. transurethral resection of prostate (TURP) in men with 30-80 cc prostates (WATER trial), Aquablation demonstrated non-inferior symptom relief as compared to TURP, but with considerable reduction in resection time and a lower risk of sexual dysfunction[19]. Notably, Aquablation was associated with a superior symptom relief benefit in a subgroup of larger (50-80 cc) prostates. Such results thus served as the impetus for the WATER II trial, a single-arm prospective, multicenter, international clinical trial of Aquablation for the surgical treatment of LUTS/BPH associated with large prostate volumes (80-150 cc). Data demonstrating 1-month feasibility and safety were recently published[20]. Herein, we present longer 3-month safety and efficacy data from the cohort of Canadian men included in the WATER II trial.

\section{Methods}

\section{Study design}

WATER II (NCT03123250) is a prospective, multicenter, international clinical trial of the Aquablation system for the treatment of BPH in men 45 to 80 years of age with a transrectal ultrasound measured prostate volume between 80 and 150 cc[20]. Only patients from Canada were included for this analysis. Inclusion criteria for this study were baseline International Prostate Symptom Score (IPSS) $\geq 12$, a maximum urinary flow rate (Qmax) $<15 \mathrm{~mL} / \mathrm{s}$, a serum creatinine $<2 \mathrm{mg} / \mathrm{dL}$, a history of failed medical therapy and mental capacity to participate in the study. Exclusion criteria were body mass index $\geq 42 \mathrm{~kg} / \mathrm{m} 2$, history of prostate or bladder cancer, clinically significant bladder calculus or bladder diverticulum, cystitis, previous urinary tract surgery, urinary catheter use daily for 90 or more consecutive days, chronic pelvic pain, urethral stricture, meatal stenosis or bladder neck contracture, use of anticholinergic agents specifically for bladder problems, and any other conditions that could prevent adequate follow-up. Patients were not excluded for prior prostate surgery or if in retention unless the catheter was in place for more than 90 days. The study was performed with Institutional Review Board approval from each participating institution and all participants provided informed consent.

\section{Study parameters}

At baseline, subjects completed IPSS[21] as well as several validated questionnaires (Incontinence Severity Index, Pain Intensity Scale, International Index of Erectile Function (IIEF-5)[22], the Male Sexual Health Questionnaire (MSHQ-EjD)[23]), uroflowmetry and postvoid residual (PVR) volume measurements, and underwent standard laboratory blood assessment. Questionnaires, uroflowmetry, PVR and laboratory tests were also required at postoperative visits at 1 and 3 months. Adverse events were recorded and rated by the clinical 
events committee as possibly, probably or definitely related to the study procedure classified as Clavien-Dindo (CD)[24] grades through a minimum of 3 months post treatment.

Aquablation technique and postoperative care

Aquablation was performed using the AquaBeam System (PROCEPT BioRobotics, Redwood City, California, USA), as described previously[19]. Following the Aquablation treatment, the bladder was thoroughly irrigated to remove residual prostate tissue and blood clots. Hemostasis was achieved using tissue tamponade with a low-pressure Foley balloon catheter which was inflated with 40-80cc of water. In addition, a tension free traction device was used to maintain traction for 4-20 hrs post procedure. If bleeding was a concern the balloon was inflated within the prostatic fossa with adequate traction using transrectal ultrasound (TRUS) guidance, prior to commencing irrigation. Unique to the procedures performed in Montreal, after the steps described above the bladder was maintained under pressure and the saline bag was attached as quickly as possible to maintain bladder pressure while the outflow is plugged. The saline was stopped once the bladder was full establishing hydrostatic pressure against the bladder neck. Patients then went to recovery without continuous bladder irrigation (CBI). At approximately 45 minutes, the surgeon checked on the patient, unplugged the outflow and started CBI. Manual irrigation was performed if required. During the next 1-4 hours, nurses aggressively titrated down the CBI flow.

\section{Statistical analysis}

A standard statistical approach was used for analysis that used the Students' t-test for continuous variables and Fisher's test for ordinal/binary variables. All statistical analysis was performed using R[25]. P value $\leq 0.05$ was considered statistically significant. Additional analysis, such as a Canada only cohort, was allowed per protocol.

\section{Results}

Procedural outcomes

Of 23 screened patients, 19 subjects (19\% of subjects enrolled in Water II) who met inclusion and exclusion criteria were enrolled at 3 Canadian academic sites between November and December 2017. Of those, 18 subjects completed the 3-month follow-up visit. All three sites had no prior Aquablation experience. Baseline characteristics are summarized in table 1. Mean age was $66.0 \pm 6.9$ years and mean baseline IPSS score was 21.2 points \pm 5.5 . Study procedures were performed under spinal anesthesia. Prostate volume ranged from 80-148 cc (consistent with study eligibility criteria) with a mean volume of $105.6 \pm 16.6$ cc. A middle lobe was present in $74 \%$ of cases with an average protrusion distance of $1.5 \mathrm{~cm} \pm 0.5$.

Mean operative time, defined as handpiece placement until final urinary catheter placement, was 33.7 \pm 7.8 minutes. The mean Aquablation resection time was 9.0 \pm 2.4 minutes. The number of men requiring a single pass, 2 passes, and greater than 2 passes during resection were $26 \%, 68 \%$, and $5 \%$, respectively. None of the patients in this cohort necessitated postAquablation cautery for hemostasis. The average length of stay following the procedure was 
$1.3 \pm 0.8$ days. At the Montreal site, 2 patients went home the same day of surgery. The average duration to final catheter removal was $2.1 \pm 1.5$ days. Hemoglobin levels decreased from a mean of $14.6 \pm 1.5 \mathrm{~g} / \mathrm{dL}$ at baseline to $12.2 \pm 1.8 \mathrm{~g} / \mathrm{dL}$ postoperatively $(\mathrm{p}<0.0001)$.

\section{Clinical endpoints}

Mean pre-, 1 month and 3 months post-treatment IPSS scores were 21.2 $\pm 5.5,9.9 \pm 6.9$ $(\mathrm{p}<0.0001)$, and $5.0 \pm 4.5(\mathrm{p}<0.0001)$. Mean pre-, 1 month and 3 months post-treatment IPSS QOL scores were 4.3 $\pm 1.2,2.1 \pm 1.9(\mathrm{p}<0.0001)$, and $1.5 \pm 2.1(\mathrm{p}<0.0001)$. Mean pre-, 1 month and 3 months post-treatment Qmax were $6.6 \pm 3.1 \mathrm{ml} / \mathrm{s}, 19.5 \pm 6.1 \mathrm{ml} / \mathrm{s}(\mathrm{p}<0.0001)$, and $23.1 \pm 9.2 \mathrm{ml} / \mathrm{s}$ $(\mathrm{p}<0.0001)$. Mean pre-, 1 month and 3 months post-treatment PVR's were 174.9 \pm 142.9 cc, 48.9 $\pm 42.2 \mathrm{cc}(\mathrm{p}<0.0001)$, and $77.7 \pm 73.6 \mathrm{cc}(\mathrm{p}<0.0001)$. Outcomes are summarized in figure 1 . Finally, at 3 months, average prostate volume decreased from 106 cc to 59 cc.

\section{Treatment-related adverse events}

The CD grade 2 or higher event rate at 3 months was 31.6\% (6 events). This was comprised of five voiding issue events (frequency, urgency, or dysuria) and one urinary tract infection. Two of these cases were CD 3a which were cystoscopies for urinary frequency and dysuria.

There were no reports of blood transfusions. The CD grade 1 persistent events consisted of ejaculatory dysfunction (32\%). There were no reported cases of incontinence $(0 \%)$ or erectile dysfunction (0\%).

\section{Discussion}

Our findings demonstrate that Aquablation of the prostate is a practical and viable option for the treatment of large sized prostate glands $(80-150 \mathrm{cc})$. The Canadian subset of patients, within this prospective multicenter Aquablation WATER II trial provides several noteworthy findings. First, 19 of $19(100 \%)$ cases were successfully completed for prostates >100 cc on average. No case was aborted, no case required cautery for hemostasis and no secondary procedures were necessary.

Second, total operative time and total resection time was 33.7 mins and 9 mins respectively on average for men with a mean prostate volume of 106 cc. Comparatively, the average time to perform a 100 cc prostate done via open prostatectomy is 95 minutes[26], via HoLEP is 91 minutes[27] and done via PVP is 93 minutes[11]. It should also be noted that this was the first experience for each institution and each surgeon with the Aquablation system and therefore, the total operative time should get shorter with greater surgical experience and proficiency with technique and instrumentation.

Third, IPSS and Qmax at 1 and 3 months post-operatively improved significanly and incrementaly from pre-operative baseline. These clinical improvements (IPSS reduced from 21 to 5 and Qmax increased from 6.6 to $23 \mathrm{ml} / \mathrm{s}$ ) are comparable to those found with HoLEP (IPSS drop from 20 to 5.3 and Qmax increases from 8.4 to $22.7 \mathrm{ml} / \mathrm{s}$ [28]) and PVP (IPSS drop from 23 to 6 and Qmax increases from 6 to $16 \mathrm{ml} / \mathrm{s}[11]$ ). Additionally, 2/3 of patients maintained antegrade ejacuation. Unless ejaculatory duct sparing is performed the rate of antegrade ejaculation preservation is very low compared to open prostatectomy, HoLEP and PVP. 
Fourth, and most importantly complications associated with this procedure were uncommon and low grade. Additionally, no patient has required further surgical treatment and no transfusions have been reported. In comparison with the larger study population, no patient in this cohort required a blood transfusion. This is most likely due to the post operative procedure care, as highligted in the methods section of this manuscript. This is particulairly important when comparing Aquablation to simple open prostatectomy for which transfusion rates have been found to be between $7-24 \%[9,10]$.

Finally, length of stay following the Aquablation procedure was $1.3 \pm 0.8$ days which is very comparable to HoLEP (1-1.3 days[15, 29]) and PVP (1-2 days[30]) and much shorter than open simple prostatectomy (3-7 days[9, 31]). In addtion, at the Montreal site 2 patients were sent home the same day of the procedure with catheter removal 24-48 hrs later. Further, experience with the Aquablation system and post-operative optimization should lead to more patients being discharged in a shorter amount of time and it is plausible that this procedure could become an outpatient procedure (day surgery).

Despite its merits, this study is not devoid of limitations. One of the limitations is that this study was a non-randomized single arm study. The impact of the study would have been greater if the study compared Aquablation to HoLEP, PVP or open prostatectomy. In addtition, the cohort was small and surgeon's experience was limited. Finally, and most importantly, the follow-up of this cohort is only 3 months. Longer term followup will be necessary to demonstrate the durability of the treatement outcomes.

The landscape of BPH surgical treatment has changed significantly over the past decade. It is a very exciting time with numerous surgical options. Smaller prostate glands ( $<50 \mathrm{cc}$ ) can be treated by almost any modality and newer office based modalities have begun to dominate this patient subpopulation. Medium sized prostate glands (50-80 cc) require more innovative techniques such as HoLEP or PVP as well as possible office based modalities. Large sized glands (>80 cc) are the most challenging and are currently managed with OSP and HoLEP and if the gland is not too large ( $<100 \mathrm{cc}$ ) then TURP or PVP are also options. Unfortunately, HoLEP is an ultraspecialized technique that only a handful of urologists can perform in Canada. This translates into long waiting times for surgery or more OSPs being performed. Aquablation is now an option for this patient subpopulation with both FDA and Health Canada approval and the updated CUA BPH guidelines have also included Aquablation as an option for surgical management of LUTS due to bladder outlet obstruction[4].

In Canada, with a uniquely socialized medical system and budget-dependent volumelimited structure to limit costs, operating room time is at a premium and consequently must be used as efficently as possible. Therefore, an effective treatment that cuts operating room time by more than $50 \%$ should be considered a major advantage. Moreover, a treatment that is ultrasound guided and robotically-executed under precise and reproducible parameters should be considered standard of care. In the age of health care reform, Canadian surgical treatment of BPH with $>20,000$ TURPs performed annually[32] should be scrutinized to maximize outcomes and 
minimize both individual treatment cost as well as patient lifetime BPH medical/surgical costs (annual dual therapy cost of $1000 \$$ year coupled with repeat TURP surgical rate of 5-10\%)).

\section{Conclusion}

In this short-term 3-month analysis of Canadian men, Aquablation appears to provide a strong surgical alternative in patients with LUTS/BPH due to larger prostate volumes with impressive functional outcomes, and relatively short operative time and length of hospital stay, and acceptable complication and low transfusion rates. The recently developed CUA guidelines list aquablation as an alternative and effective surgical option for patients suffering from LUTS due to moderate/large sized prostate volumes. This precise ultrasound guided, robotically executed prostatic ablative procedure provides excellent clinical outcomes with minimal complications. Longer-term followup is ongoing to confirm these results. 


\section{References}

1. Wei, J.T., E. Calhoun, and S.J. Jacobsen, Urologic diseases in America project: benign prostatic hyperplasia. J Urol, 2005. 173(4): p. 1256-61.

2. McVary, K.T., BPH: epidemiology and comorbidities. Am J Manag Care, 2006. 12(5 Suppl): p. S122-8.

3. Roehrborn, C.G., et al., The effects of combination therapy with dutasteride and tamsulosin on clinical outcomes in men with symptomatic benign prostatic hyperplasia: 4-year results from the CombAT study. Eur Urol, 2010. 57(1): p. 123-31.

4. Nickel JC, A.L., Barkin J, Elterman D, Nachabe M, Zorn KC, Male Lower Urinary Tract Symptoms/Benign Prostatic Hyperplasia (MLUTS/BPH) Canadian Urological Association Guidelines: 2018 Update. Canadian Urological Association Journal, 2018. in press.

5. McVary, K.T., et al., Update on AUA guideline on the management of benign prostatic hyperplasia. J Urol, 2011. 185(5): p. 1793-803.

6. Gratzke, C., et al., EAU Guidelines on the Assessment of Non-neurogenic Male Lower Urinary Tract Symptoms including Benign Prostatic Obstruction. Eur Urol, 2015. 67(6): p. 1099-1109.

7. Varkarakis, I., et al., Long-term results of open transvesical prostatectomy from a contemporary series of patients. Urology, 2004. 64(2): p. 306-10.

8. Lin, Y., et al., Transurethral enucleation of the prostate versus transvesical open prostatectomy for large benign prostatic hyperplasia: a systematic review and metaanalysis of randomized controlled trials. World J Urol, 2016. 34(9): p. 1207-19.

9. Pariser, J.J., et al., National Trends of Simple Prostatectomy for Benign Prostatic Hyperplasia With an Analysis of Risk Factors for Adverse Perioperative Outcomes. Urology, 2015. 86(4): p. 721-5.

10. Gratzke, C., et al., Complications and early postoperative outcome after open prostatectomy in patients with benign prostatic enlargement: results of a prospective multicenter study. J Urol, 2007. 177(4): p. 1419-22.

11. Valdivieso, R., et al., Multicentre international experience of 532-nm laser photoselective vaporization with GreenLight XPS in men with very large prostates. BJU Int, 2018.

12. Hueber, P.A., et al., Photoselective Vaporization of the Prostate for Benign Prostatic Hyperplasia Using the 180 Watt System: Multicenter Study of the Impact of Prostate Size on Safety and Outcomes. J Urol, 2015. 194(2): p. 462-9.

13. Naspro, R., et al., Holmium laser enucleation of the prostate versus open prostatectomy for prostates $>70$ g: 24-month follow-up. Eur Urol, 2006. 50(3): p. 563-8.

14. Gilling, P.J., et al., Long-term results of a randomized trial comparing holmium laser enucleation of the prostate and transurethral resection of the prostate: results at 7 years. BJU Int, 2012. 109(3): p. 408-11. 
15. El Tayeb, M.M., et al., Holmium Laser Enucleation of the Prostate in Patients Requiring Anticoagulation. J Endourol, 2016. 30(7): p. 805-9.

16. Elzayat, E.A. and M.M. Elhilali, Holmium laser enucleation of the prostate (HoLEP): long-term results, reoperation rate, and possible impact of the learning curve. Eur Urol, 2007. 52(5): p. 1465-71.

17. Robert, G., et al., Multicentre prospective evaluation of the learning curve of holmium laser enucleation of the prostate (HoLEP). BJU Int, 2016. 117(3): p. 495-9.

18. Gilling, P., P. Anderson, and A. Tan, Aquablation of the Prostate for Symptomatic Benign Prostatic Hyperplasia: 1-Year Results. J Urol, 2017. 197(6): p. 1565-1572.

19. Gilling, P., et al., WATER: A Double-Blind, Randomized, Controlled Trial of Aquablation((R)) vs Transurethral Resection of the Prostate in Benign Prostatic Hyperplasia. J Urol, 2018. 199(5): p. 1252-1261.

20. Desai, M., et al., Aquablation Procedural Outcomes for BPH in Large Prostates (80150cc): Initial Experience. BJU Int, 2018.

21. Barry, M.J., et al., The American Urological Association symptom index for benign prostatic hyperplasia. The Measurement Committee of the American Urological Association. J Urol, 1992. 148(5): p. 1549-57; discussion 1564.

22. Rosen, R.C., et al., Minimal clinically important differences in the erectile function domain of the International Index of Erectile Function scale. Eur Urol, 2011. 60(5): p. 1010-6.

23. Rosen, R.C., et al., Male Sexual Health Questionnaire (MSHQ): scale development and psychometric validation. Urology, 2004. 64(4): p. 777-82.

24. Dindo, D., N. Demartines, and P.A. Clavien, Classification of surgical complications: a new proposal with evaluation in a cohort of 6336 patients and results of a survey. Ann Surg, 2004. 240(2): p. 205-13.

25. R Core Team: A Language and Environment for Statistical Computing [Internet]. Vienna, Austria: R Foundation for Statistical Computing. 2016; Available from: https://www.R-project.org/.

26. Sorokin, I., et al., Robot-Assisted Versus Open Simple Prostatectomy for Benign Prostatic Hyperplasia in Large Glands: A Propensity Score-Matched Comparison of Perioperative and Short-Term Outcomes. J Endourol, 2017. 31(11): p. 1164-1169.

27. Monn, M.F., et al., Predictors of Enucleation and Morcellation Time During Holmium Laser Enucleation of the Prostate. Urology, 2015. 86(2): p. 338-42.

28. Krambeck, A.E., S.E. Handa, and J.E. Lingeman, Experience with more than 1,000 holmium laser prostate enucleations for benign prostatic hyperplasia. J Urol, 2010. 183(3): p. 1105-9.

29. Bhojani, N., et al., Coexisting prostate cancer found at the time of holmium laser enucleation of the prostate for benign prostatic hyperplasia: predicting its presence and grade in analyzed tissue. J Endourol, 2015. 29(1): p. 41-6. 
30. Meskawi, M., et al., Multicenter international experience of 532 nm-laser photovaporization with Greenlight XPS in men with large prostates (prostate volume $>100 \mathrm{cc}$ ). World J Urol, 2017. 35(10): p. 1603-1609.

31. Misrai, V., et al., Comparison between open simple prostatectomy and green laser enucleation of the prostate for treating large benign prostatic hyperplasia: a single-centre experience. World J Urol, 2018. 36(5): p. 793-799.

32. Hueber, P.A. and K.C. Zorn, Canadian trend in surgical management of benign prostatic hyperplasia and laser therapy from 2007-2008 to 2011-2012. Can Urol Assoc J, 2013. 7(9-10): p. E582-6. 


\section{Figures and Tables}

Fig. 1. One- and three-month functional outcomes following Aquablation in 19 men with lower urinary tract symptoms/benign prostatic hyperplasia and large prostates. (A) International Prostate Symptom Score (IPSS); (B) IPSS quality of life (QOL); (C) maximum urinary flow rate (Qmax); (D) post-void residual (PVR). * Statistical significance compared to baseline. ${ }^{++}$Statistical significance compared to one month. CI: confidence interval.

A. Total IPSS

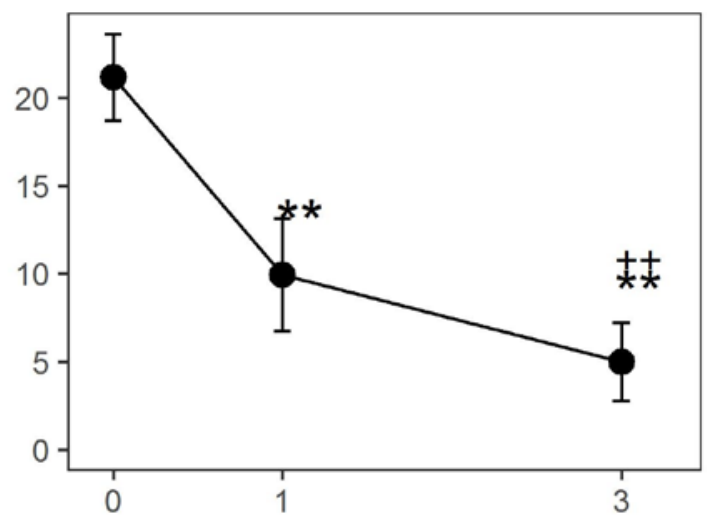

C. Qmax

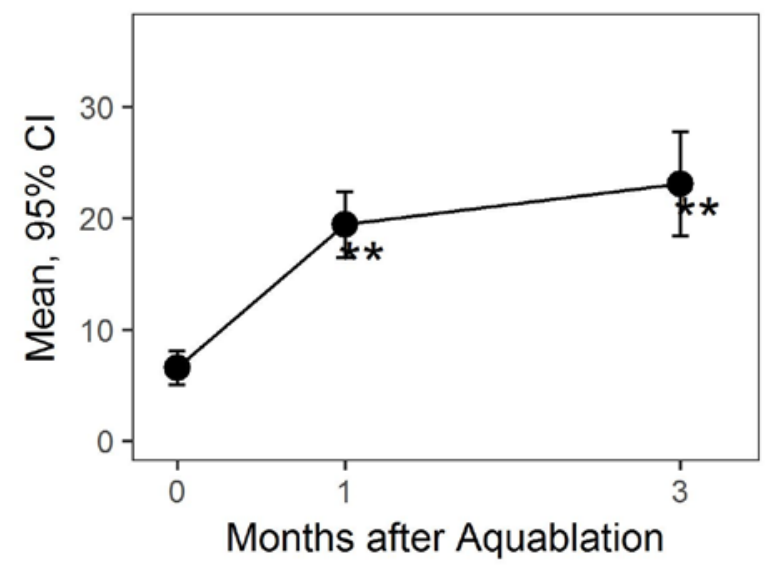

B. IPSS QOL

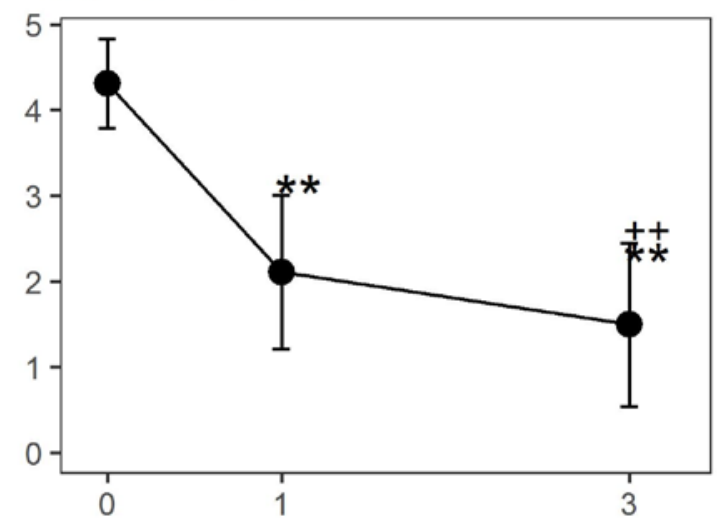

D. PVR

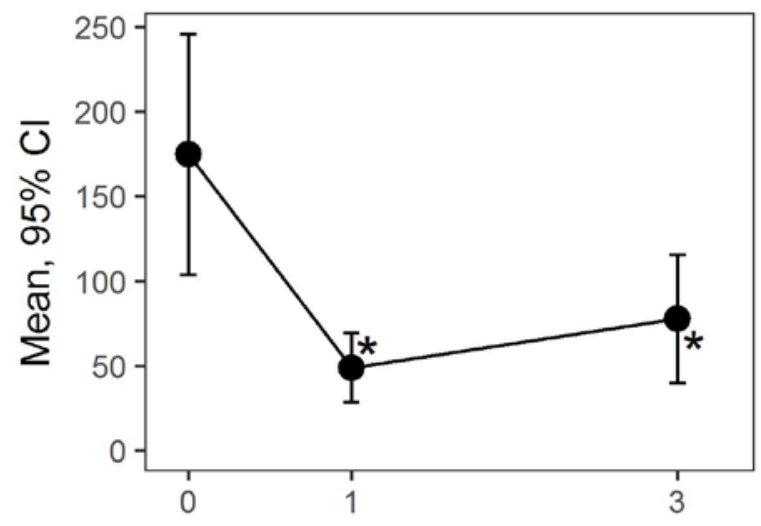




\begin{tabular}{|l|c|}
\hline Table 1. Baseline characteristics (n=19) \\
\hline Characteristic & Statistic, mean (SD) \\
\hline Age, years, mean (SD) & $66(6.9)$ \\
\hline Body mass index, mean (SD) & $27.6(2.9)$ \\
\hline Prostate-specific antigen, g/dL; mean (SD) & $10.3(9.3)$ \\
\hline Use of catheters in 45 days prior to enrollment & $12(21.1 \%)$ \\
\hline Prostate size (TRUS), cc; mean (SD) & $105.6(16.6)$ \\
\hline Middle lobe & $74 \%$ \\
\hline Intravesical component & $100 \%$ \\
Intravesical protrusion, cm; mean (SD) & $1.5(0.5)$ \\
\hline Baseline questionnaires & $21.2(5.5)$ \\
\hline IPSS score, mean (SD) & $4.3(1.2)$ \\
\hline IPSS QOL, mean (SD) & $16(84.2 \%)$ \\
\hline Sexually active, n (\%) (MSHQ-EjD) & $8.3(3)$ \\
\hline MSHQ-EjD mean (SD) & $15.5(7.4)$ \\
\hline IIEF-5, mean (SD) & \\
\hline
\end{tabular}

IIEF: International Index of Erectile Function; IPSS: International Prostate Symptom Score; MSHQ-EjD: Male Sexual Health Questionnaire - ejaculatory dysfunction; QOL: quality of life; SD: standard deviation; TRUS: transrectal ultrasound. 